\title{
AC transport in Correlated Quantum Dots: From Kondo to Coulomb blockade regime
}

\author{
G. Stefanucci ${ }^{1,2}$ and S. Kurth ${ }^{3,4}$ \\ ${ }^{1}$ Dipartimento di Fisica, Università di Roma Tor Vergata, Via della Ricerca Scientifica 1, \\ 00133 Rome, Italy; European Theoretical Spectroscopy Facility (ETSF) \\ ${ }^{2}$ INFN, Laboratori Nazionali di Frascati, Via E. Fermi 40, 00044 Frascati, Italy \\ ${ }^{3}$ Nano-Bio Spectroscopy Group and European Theoretical Spectroscopy Facility (ETSF), \\ Dpto. de Física de Materiales, Universidad del País Vasco UPV/EHU, Av. Tolosa 72, E-20018 San Sebastián, Spain \\ ${ }^{4}$ IKERBASQUE, Basque Foundation for Science, Maria Diaz de Haro 3, E-48013 Bilbao, Spain
}

\begin{abstract}
We explore the finite bias DC differential conductance of a correlated quantum dot under the influence of an AC field, from the low-temperature Kondo to the finite temperature Coulomb blockade regime. Real-time simulations are performed using a time-dependent generalization of the steadystate density functional theory (i-DFT) [Nano Lett. 15, 8020 (2015)]. The numerical simplicity of i-DFT allows for unprecedented long time evolutions. Accurate values of average current and density are obtained by integrating over several periods of the AC field. We find that (i) the zerotemperature Kondo plateau is suppressed, (ii) the photon-assisted conductance peaks are shifted due to correlations and (iii) the Coulomb blockade is lifted with a concomitant smoothening of the sharp diamond edges.
\end{abstract}

Over the last decades there have been tremendous advances in manufacturing nanoscale devices such as nanotubes, artificial atoms (quantum dots), or single molecules attached to metallic leads $1-4]$. The theoretical description of their transport properties often remains a challenge since it involves the physics of strong correlations out of thermal equilibrium. Even the (minimal) single impurity Anderson model [5] (SIAM), after almost sixty years is still a matter of intense research and debate. The development of clever analytical and numerical techniques has provided us with a fairly good understanding of its equilibrium and steady state properties. Unfortunately, the same cannot be said for timedependent responses. In particular, very little is known about the behavior of the average current when an $\mathrm{AC}$ field is superimposed to a DC voltage. What is the fate of the zero-temperature Kondo plateau in the conductance? How does the charging energy affect the photon-assisted conductance peaks? Is the finite-temperature Coulomb blockade lifted? In this Letter we provide an answer to these and related fundamental questions.

One way to access AC (linear as well as nonlinear) transport properties consists in performing numerical simulations of the time-evolution of the system. Several time-propagation algorithms have been put forward. Time-dependent (TD) density matrix renormalization group 6 -10], TD numerical renormalization group 11], functional renormalization group [12 14, real-time Monte Carlo 15 19], hierarchical equations of motion approach 20], iterative real-time path integral 21] and real-time effective action 22] have all been used to investigate the transient response to a sudden quench of bias or gate voltages, and a reasonable agreement between them has been reached. These methods, however, are limited to short propagation times and AC responses are difficult to address since convergence often requires averaging the TD current over several periods of the driving field. An alternative to time-propagation is the Floquet Green's function approach. SIAM responses to an oscillating gate have been reported in Refs. [23, 24] using either a second-order self-energy (reasonable only at the particle-hole symmetric point) or an analytically interpolated equilibrium self-energy (questionable for AC voltages). To the best of our knowledge, no attempts to calculate the SIAM conductance under AC voltages have been made so far.

Lately, strongly correlated systems have been studied also with density functional theory (DFT) both in its static 25 29] and time-dependent version 30 35]. In a recent work we proposed a steady-state density functional theory [36] (i-DFT) to calculate the steady density on and the steady current through a quantum junction sandwiched between metallic leads. In Ref. [37] we applied i-DFT to the SIAM and found excellent agreement with numerically exact methods in a wide range of bias and gate voltages, from weak to strong charging energies $U$ and from low to high temperatures $T$.

We here extend i-DFT to the time domain, thereby laying down a computationally feasible scheme to shed light on the AC transport properties of the SIAM. Our main findings are (i) AC voltage suppression of the $T=0$ Kondo plateau (ii) interaction-induced shift of the photon-assisted conductance peaks and (iii) lifting of the finite-temperature Coulomb blockade with concomitant smoothening of the diamond shape.

Time-Dependent $i$-DFT - i-DFT establishes a one-toone correspondence between the pair density on and current through the impurity, $(n, I)$, and the pair gate and bias voltages $(v, V)$. Accordingly, there exists a unique pair $\left(v_{s}, V_{s}\right)$ that in the noninteracting SIAM produces the same density and current as in the interacting one. The pair $\left(v_{s}, V_{s}\right)$ consists of the Kohn-Sham (KS) gate $v_{s}=v+v_{\mathrm{H}}+v_{\mathrm{xc}}$ and $\mathrm{KS}$ voltage $V_{s}=V+V_{\mathrm{xc}}$ where the Hartree potential $v_{\mathrm{H}}=U n$ whereas the exchange- 
correlation (xc) potentials $v_{\mathrm{xc}}$ and $V_{\mathrm{xc}}$ are universal functionals (i.e., independent of the external fields $v$ and $V$ ) of both density and current. Knowledge of these functionals allows for calculating $(n, I)$ by solving self-consistently the equations

$$
\begin{gathered}
n=2 \sum_{\alpha=L, R} \int \frac{d \omega}{2 \pi} f\left(\omega-V_{s, \alpha}\right) A_{\alpha}(\omega) \\
I=2 \int \frac{d \omega}{2 \pi}\left[f\left(\omega-V_{s, L}\right)-f\left(\omega-V_{s, R}\right)\right] \mathcal{T}(\omega)
\end{gathered}
$$

where $V_{s, L}=-V_{s, R}=V_{s} / 2$ is the bias applied to the left $(L)$ and right $(R)$ leads and $f(\omega)=1 /\left(e^{\beta(\omega-\mu)}+1\right)$ is the Fermi function at inverse temperature $\beta=1 / T$ and chemical potential $\mu$. In Eq. (11) the partial spectral function is given by $A_{\alpha}(\omega) \equiv G(\omega) \Gamma_{\alpha}(\omega) G^{\dagger}(\omega)$ with the (retarded) KS Green's function $G(\omega)=1 /\left(\omega-v-v_{\mathrm{Hxc}}-\right.$ $\left.\Sigma_{L}(\omega)-\Sigma_{R}(\omega)\right)$ and broadening $\Gamma_{\alpha}(\omega)=-2 \operatorname{Im}\left[\Sigma_{\alpha}(\omega)\right]$, $\Sigma_{\alpha}$ being the embedding self-energy of lead $\alpha$. The current in Eq. (2) is expressed in terms of the KS transmission coefficient $\mathcal{T}(\omega)=A_{L}(\omega) \Gamma_{R}(\omega)$. The self-consistent solution of Eqs. (11) and (2) is extremely efficient [37, 38]. It is therefore natural to extend i-DFT to the time domain and explore dynamical (as opposed to steadystate) transport properties. Setting the external bias $V(t)=\theta(t)\left[V+V_{\mathrm{AC}} \sin (\Omega t)\right]$ we can provide a full characterization of the $\mathrm{AC}$ conductance.

In the same spirit of the adiabatic approximations in standard TD-DFT [39, 40] we calculate the TD density $n(t)$ and current $I(t)$ by propagating the KS SIAM with TD gate potential $v_{s}(t)=v(t)+v_{\mathrm{Hxc}}[n(t), I(t)]$ and voltage $V_{s}(t)=V(t)+V_{\mathrm{xc}}[n(t), I(t)]$. The propagation is performed by solving the coupled TD KS equation

$$
i \frac{d}{d t}\left(\begin{array}{l}
\psi_{L, k} \\
\psi_{C, k} \\
\psi_{R, k}
\end{array}\right)=\left(\begin{array}{ccc}
h_{L L} & h_{L C} & 0 \\
h_{C L} & h_{C C} & h_{C R} \\
0 & h_{R C} & h_{R R}
\end{array}\right)\left(\begin{array}{l}
\psi_{L, k} \\
\psi_{C, k} \\
\psi_{R, k}
\end{array}\right)
$$

for every KS state $\psi_{k}$. Equation (3) is written in a block form where the blocks $L$ and $R$ refer to the left and right leads whereas the block $C$ refers to the impurity site, hence $h_{C C}=v_{s}(t)$. We model the leads as semi-infinite tight-binding chains with nearest neighbor hopping $t_{\text {lead }}$ and onsite energy $V_{s, \alpha}(t)$. Only the boundary site of the semi-infinite chains is connected to the impurity and the corresponding hopping amplitude is $t_{\text {link }}$. We take the leads at half-filling $(\mu=0)$, choose both $t_{\text {lead }}$ and $t_{\text {link }}$ much larger than any other energy scale and set the ratio $t_{\text {link }}^{2} / t_{\text {lead }}=\gamma / 2$ to stay in the wide band limit (WBL).

Equation (3) is solved using a generalization of the algorithm of Ref. 41 to finite temperatures. The time propagation is performed with a predictor-corrector scheme at each time step for $v_{\mathrm{Hxc}}$ and $V_{\mathrm{xc}}$. The TD occupation and current to plug into the xc potentials are obtained from the KS wavefunctions according to

$$
n(t)=2 \sum_{k} f\left(\epsilon_{k}\right)\left|\psi_{C, k}(t)\right|^{2},
$$

$$
I(t)=\frac{I_{L}(t)-I_{R}(t)}{2} .
$$

The current at the $\alpha$ interface

$$
I_{\alpha}(t)=4 \sum_{k} f\left(\epsilon_{k}\right) \operatorname{Im}\left[t_{\text {link }} \psi_{\alpha, k}^{*}(t) \psi_{C, k}(t)\right]
$$

is expressed in terms of the wavefunction $\psi_{\alpha, k}(t)$ at boundary site of lead $\alpha$. Notice that current conservation implies $I_{L}(t)+I_{R}(t)+\dot{n}(t)=0$ at all times. For symmetric voltages $V_{L}(t)=-V_{R}(t)=V(t) / 2$ and at the particle-hole symmetric point (ph-SP) $v=-U / 2$ we have $I_{L}(t)=-I_{R}(t)$.

In Ref. 37] we proposed an accurate parametrization of $v_{\mathrm{Hxc}}$ and $V_{\mathrm{xc}}$. This parametrization has been shown to agree well with results from the functional and numerical renormalization group in a wide range of temperatures and interaction strengths. We have used the xc potentials of Ref. [37] to solve Eq. (3). A somewhat related currentdependent approximation for $v_{\mathrm{Hxc}}$, valid for only one lead and at temperatures higher than the Kondo temperature, has recently been suggested in Ref. 42 .

Results - We consider the SIAM initially (times $t \leq 0$ ) in thermal equilibrium and then driven out of equilibrium by a symmetric bias $V_{L}(t)=-V_{R}(t)=V(t) / 2$. The bias is the sum of a DC and an AC contribution, i.e.,

$$
V(t)=V+V_{\mathrm{AC}} \sin (\Omega t) \quad \text { for } t>0 .
$$

After a sufficiently long propagation time $t_{\ell}$ the transient features die out and all physical observables become periodic functions of time. We then calculate the DC component of the current by averaging $I(t)$ over $N$ periods $\tau=2 \pi / \Omega$, i.e., $I_{\mathrm{DC}}=\int_{t_{\ell}}^{t_{\ell}+N \tau} I(t) /(N \tau)$. Depending on the parameters, a good convergence may require $N$ of the order of 10 or larger. Finally we calculate the finite-bias DC conductance $G=\mathrm{d} I_{\mathrm{DC}} / \mathrm{d} V$, highlighting its most relevant features as $V_{\mathrm{AC}}$ and $\Omega$ are varied. We consider over two physically distinct regimes: the Kondo regime at zero temperature and the Coulomb blockade $(\mathrm{CB})$ regime at temperatures larger than the Kondo temperature.

First we checked that for $U=V=0$ our TD algorithm agrees with the exact (in the WBL approximation) noninteracting formula [43]

$$
G^{U=0}=\sum_{k=-\infty}^{\infty} J_{k}^{2}\left(-\frac{V_{\mathrm{AC}}}{\Omega}\right) \frac{\frac{\gamma^{2}}{4}}{(v-k \Omega)^{2}+\frac{\gamma^{2}}{4}}
$$

where $J_{k}(x)$ is the Bessel function of order $k$. Without interactions photon-assisted transport (PAT), i.e., charge transfer accompanied by the emission or absorption of photons, is the only scattering mechanism.

Turning on the interaction the scenario changes dramatically, especially for gates $v$ in the range $(-U, 0)$ where correlation effects are enhanced. In Fig. 1 we show results for the zero-bias DC conductance as function of 


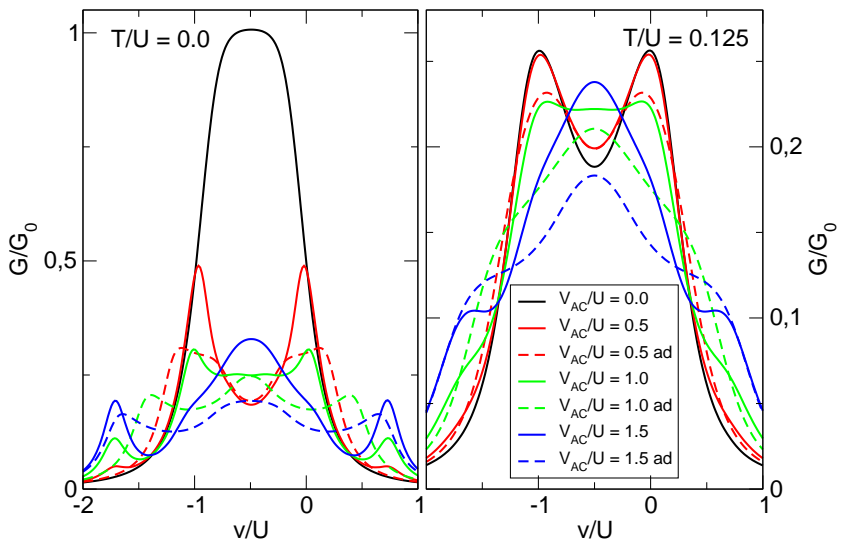

FIG. 1. Zero-bias DC conductance (solid lines) as well as its adiabatic counterpart of Eq. (9) (dashed) versus $v$ for different AC amplitudes. The SIAM parameters are $U / \gamma=4, \Omega / U=$ $\pi / 4$ and temperature $T=0$ (left panel) and $T / U=0.125$ (right panel). $G_{0}=\frac{1}{\pi}$ is the quantum of conductance.

$v$ for different $V_{\mathrm{AC}}$. At temperature $T=0$ (left panel) and already for small $V_{\mathrm{AC}}$ the Kondo plateau is drastically suppressed. The DC conductance resembles the one at $V_{\mathrm{AC}}=0$ in the $\mathrm{CB}$ regime with two peaks separated by roughly the charging energy $U$ and a minimum at the ph-SP. The suppression of Kondo correlations has already been observed in Refs. 44, 45] using a perturbative approach as well as in Refs. 23, 24] where, rather than an AC bias, the gate potential is perturbed harmonically. As $V_{\mathrm{AC}}$ increases, however, the minimum is first converted into a plateau and eventually into a maximum with the concomitant washing out of the side peaks. The decrease and subsequent increase of the Kondo peak at the ph-SP predicted by our calculations is in agreement with experimental findings reported in Ref. [46].

In the $\mathrm{CB}$ regime $(T / U=0.125$, right panel $)$ a small $\mathrm{AC}$ bias barely changes the values of $G$ at $V_{\mathrm{AC}}=0$. However, for $V_{\mathrm{AC}} \approx U$ the $\mathrm{DC}$ conductance develops a plateau and a maximum at the ph-SP for even larger amplitudes. In both regimes, see left and right panels, we also observe increasingly higher side peaks at $v \approx \Omega$ and $v \approx-U-\Omega$ with increasing $V_{\mathrm{AC}}$, a clear signature of PAT. We will discuss the precise position of these peaks below.

To highlight nonadiabatic (memory) effects we define the "adiabatic" DC conductance according to

$$
G_{\mathrm{ad}}=\frac{1}{\tau} \int_{0}^{\tau} \mathrm{d} t G_{\mathrm{DC}}(V(t))
$$

where $G_{\mathrm{DC}}(V)=\mathrm{d} I /\left.\mathrm{d} V\right|_{V_{\mathrm{AC}}=0}$ is the finite-bias DC conductance at vanishing $\mathrm{AC}$ amplitude. The results for $G_{\text {ad }}$ are shown in both panels of Fig. 1 (dashed lines). For small $V_{\mathrm{AC}}$ and around the ph-SP, $G_{\mathrm{ad}}$ is remarkably close to $G$ both in the Kondo and the CB regime while away from ph-SP the two quantities only share qualitative features at best. For larger $V_{\mathrm{AC}}$, adiabatic and non-

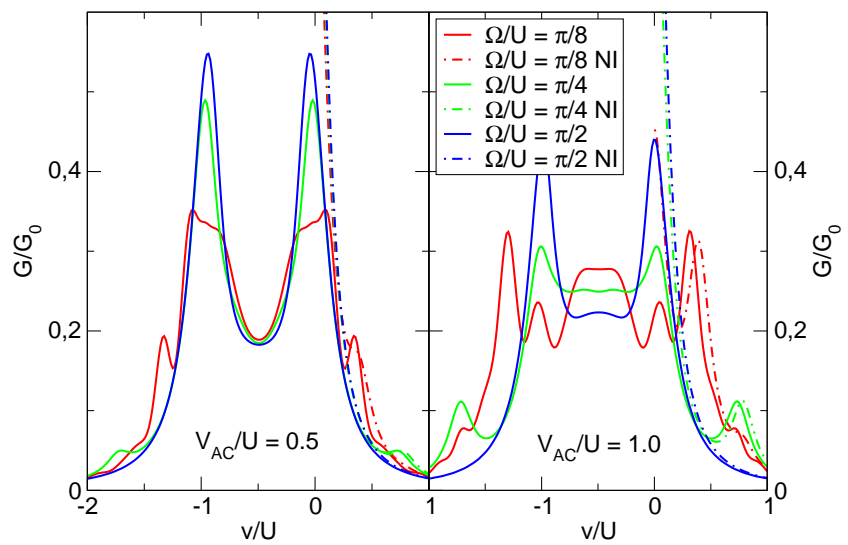

FIG. 2. Zero-bias DC conductance versus $v$ for different AC freouencies and for amnlitudes $V_{\wedge r} / J=0.5$ (left nanel) and

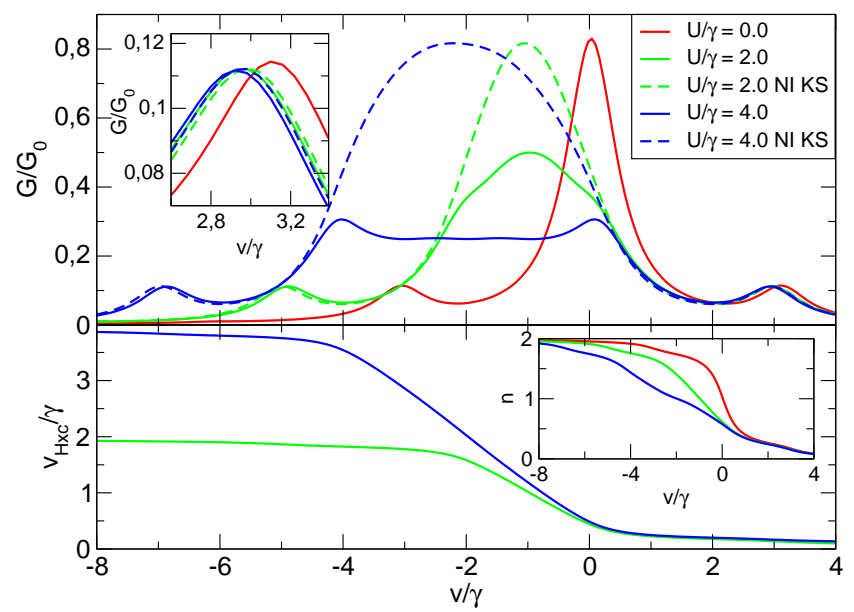

FIG. 3. Upper panel: Zero-bias DC conductances (solid) versus $v$ for $\Omega / \gamma=\pi, V_{\mathrm{AC}} / \gamma=4.0$ and different values of $U$. Dashed lines are the noninteracting result of Eq. (8) with $v$ replaced by the time-averaged KS potential $v_{s}$. Inset: zoom in around $v=\Omega$ showing shifts in the peak position of the PAT peak. Lower panel: time-averaged Hxc gate and density (inset).

adiabatic DC conductances increasingly differ, pointing to the importance of memory effects.

In Fig. 2 we show the dependence of the zero-bias $G$ at $T=0$ on the AC frequency for two different AC amplitudes. At the small amplitude $V_{\mathrm{AC}} / U=0.5$ (left panel) the behavior of $G$ is qualitatively similar for different frequencies (suppression of the Kondo plateau and arising of PAT peaks). Interestingly, for the larger amplitude $V_{\mathrm{AC}} / U=1.0$ (right panel), $G$ develops a plateau around the ph-SP for all frequencies, with a height that decreases monotonically with $\Omega$. For this larger amplitude the PAT peaks emerge more distinctly and we clearly appreciate the nonlinear (in $V_{\mathrm{AC}}$ ) effect of absorption/emission of two photons (for $\Omega / U=\pi / 8$ ).

The dashed curves starting at $v=0$ in both panels 


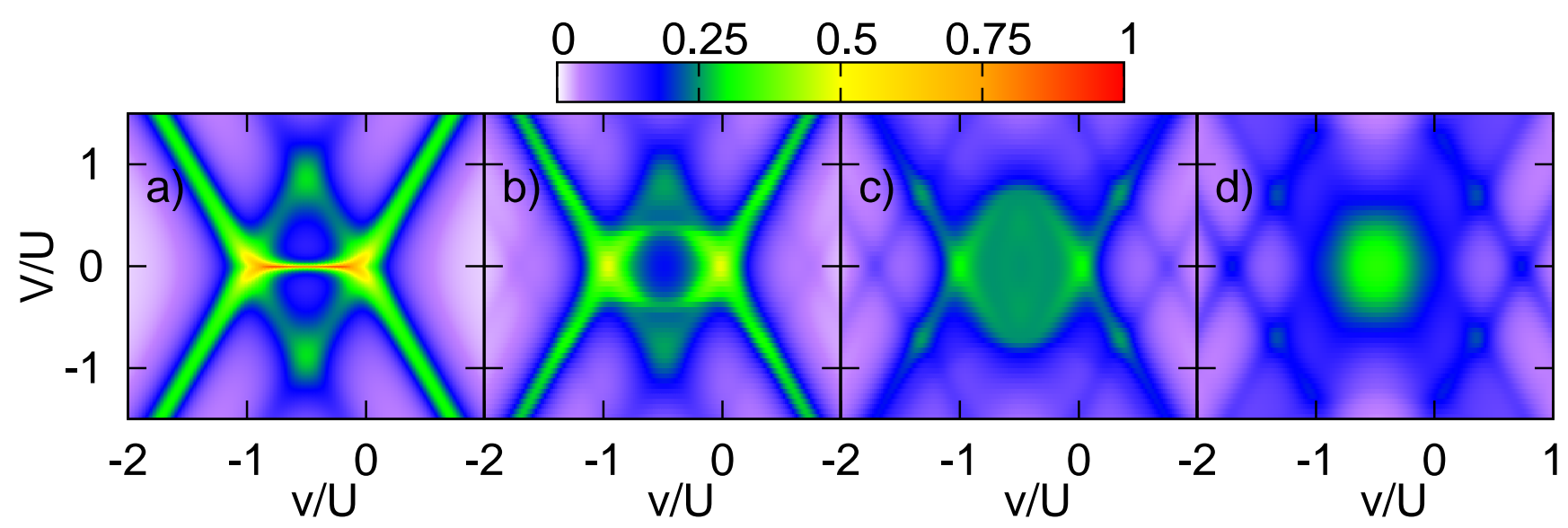

FIG. 4. DC conductance in the $(v, V)$ plane at zero temperature for a) $\left.\left.V_{\mathrm{AC}} / U=0.0, \mathrm{~b}\right) V_{\mathrm{AC}} / U=0.5, \mathrm{c}\right) V_{\mathrm{AC}} / U=1.0$ and d) $V_{\mathrm{AC}} / U=1.5$. Other parameters as in Fig. 1.

of Fig. 2 are the values of $G^{U=0}$, see Eq. (8) (which is an even functions of $v$ ), exhibiting PAT peaks at $v=$ $k \Omega$ with $k$ integer [43]. For $v>0$, the interacting and noninteracing results agree reasonably well while for $v<$ 0 correlations shift the PAT peaks by approximately $-U$.

It is worth noting that in the interacting case the PAT peak at $v \approx \Omega$ is slightly shifted to lower gates. To shed some light on this shift we calculated the zero-bias $G$ for various charging energies $U$. The results are shown in the upper panel of Fig. 3 where the inset is a magnification of the PAT peak at $v \approx \Omega$. For these gate values correlations are weak as confirmed by the small value $n \approx 0.15$ of the time-averaged density, see inset in the lower panel. In the weakly correlated regime the DC conductance is well approximated by the noninteracting formula of Eq. (8). However, from the i-DFT perspective, the $v$ in Eq. (8) is not just the bare gate but instead the time-averaged KS gate $v_{s}=v+v_{\mathrm{Hxc}}$, i.e., the sum of the bare gate and the time-averaged Hxc potential $v_{\mathrm{Hxc}}$ shown in the lower panel of Fig. 3. Consequently, the PAT peak in $G$ occurs at $v=\Omega-v_{\mathrm{Hxc}}$, i.e., it is shifted to lower energies. This is precisely the shift we observe in our calculations. In fact, if we evaluate Eq. (8) making the replacement $v \rightarrow v_{s}$ we get a remarkable good agreement with the interacting $G$ (including position and height of the PAT peaks) for $v$ outside the interval $(-U, 0)$, see dashed curves in upper panel of Fig. 3. Obviously, this good agreement breaks down for gates in the interval $(-U, 0)$ since the system can no longer be considered weakly correlated.

So far we presented results for the zero-bias DC conductance. However, the proposed method is not limited to this very special case. In Fig. 4 we display the finitebias $G$ as function of $v$ and $V$ for different amplitudes of the AC field. The calculations are done at temperature $T=0$. To appreciate the effects of the AC field the wellknown pure DC result, i.e., $V_{\mathrm{AC}}=0$, is shown in panel a). We correctly reproduce the $\mathrm{CB}$ diamond as well as the Kondo resonance at $V=0$. A moderate amplitude of the
AC field, panel b), leads to a suppression of the Kondo resonance (see also Fig. 1). Moreover, starting from the finite bias corners at $v / U=-1 / 2$ and $V / U= \pm 1$, the $\mathrm{CB}$ is lifted inside the diamond. As $V_{\mathrm{AC}}$ increases further, panels c) and d), the CB is lifted everywhere in the diamond, leaving for the largest amplitude, panel d), an area of increased conductance around the ph-SP at $V=0$. We also find that the side peaks due to PAT seen in Figs. 1 and 2 branch at finite bias into straight lines with negative and positive slopes, reminiscent of the typical $G$ lines at $V_{\mathrm{AC}}=0$. Not surprisingly, these lines become more pronounced as $V_{\mathrm{AC}}$ increases.

In summary, we have investigated electron transport through a correlated quantum dot subjected simultaneously to DC and AC biases by explicit and numerically efficient time propagation in a DFT framework. For the corresponding Hxc gate and xc bias potentials we have suggested a time-local approximation based on recently proposed accurate i-DFT functionals for the steady state. We find that in the Kondo regime already for small AC amplitude the Kondo plateau in the zero-bias DC conductance is strongly suppressed while in the $\mathrm{CB}$ regime the changes are less pronounced. The observed shift of the photon-assisted transport peaks due to electronic interactions can readily be explained within DFT. At finite DC bias, Coulomb blockade is lifted with increasing AC amplitude and the CB diamond is deformed.

G.S. acknowledges funding by MIUR FIRB Grant No. RBFR12SW0J, EC funding through the RISE Co-ExAN (GA644076), and funding through the INFN-Nemesys project. S.K. acknowledges funding by a grant of the "Ministerio de Economia y Competividad (MINECO)" (FIS2016-79464-P) and by the "Grupos Consolidados UPV/EHU del Gobierno Vasco" (IT578-13). 
[1] G. Cuniberti, G. Fagas, and K. Richter, Introducing Molecular Electronics (Springer, Heidelberg, 2005).

[2] Y. V. Nazarov and Y. M. Blanter, Quantum Transport: Introduction to Nanoscience (Cambridge University Press, Cambridge, 2009).

[3] J. C. Cuevas and E. Scheer, Molecular Electronics: An Introduction to Theory and Experiment (World Scientific, London, 2010).

[4] I. Baldea, Molecular Electronics: An Experimental and Theoretical Approach (Pan Stanford, 2015).

[5] P. W. Anderson, Phys. Rev. 124, 41 (1961)

[6] K. A. Al-Hassanieh, A. E. Feiguin, J. A. Riera, C. Büsser, and E. Dagotto, Phys. Rev. B 73, 195304 (2006)

[7] L. G. G. V. Dias da Silva, F. Heidrich-Meisner, A. E. Feiguin, C. A. Büsser, G. B. Martins, E. V. Anda, and E. Dagotto, Phys. Rev. B 78, 195317 (2008).

[8] F. Heidrich-Meisner, A. E. Feiguin, and E. Dagotto, Phys. Rev. B 79, 235336 (2009)

[9] M. Nuss, M. Ganahl, H. G. Evertz, E. Arrigoni, and W. von der Linden, Phys. Rev. B 88, 045132 (2013).

[10] S. Kirino, T. Fujii, J. Zhao, and K. Ueda, J. Phys. Soc. Jpn. 77, 084704 (2008).

[11] F. B. Anders and A. Schiller, Phys. Rev. Lett. 95, 196801 (2005).

[12] S. G. Jakobs, M. Pletyukhov, and H. Schoeller, Phys. Rev. B 81, 195109 (2010)

[13] J. Eckel, F. Heidrich-Meisner, S. G. Jakobs, M. Thorwart, M. Pletyukhov, and R. Egger, New J. Phys. 12, 043042 (2010).

[14] C. Karrasch, V. Meden, and K. Schönhammer, Phys. Rev. B 82, 125114 (2010)

[15] L. Mühlbacher and E. Rabani, Phys. Rev. Lett. 100, 176403 (2008).

[16] A. E. Antipov, Q. Dong, and E. Gull, Phys. Rev. Lett. 116, 036801 (2016).

[17] P. Werner, T. Oka, M. Eckstein, and A. J. Millis, Phys. Rev. B 81, 035108 (2010)

[18] P. Werner, T. Oka, and A. J. Millis, Phys. Rev. B 79, 035320 (2009)

[19] A. Droghetti and I. Rungger, Phys. Rev. B 95, 085131 (2017)

[20] Y. Cheng, W. Hou, Y. Wang, Z. Li, J. Wei, and Y. Yan, New J. Phys. 17, 033009 (2015).

[21] S. Weiss, J. Eckel, M. Thorwart, and R. Egger, Phys. Rev. B 77, 195316 (2008)

[22] S. Bock, A. Liluashvili, and T. Gasenzer, Phys. Rev. B 94, 045108 (2016)
[23] R. López, R. Aguado, G. Platero, and C. Tejedor, Phys. Rev. B 64, 075319 (2001).

[24] R. López, R. Aguado, G. Platero, and C. Tejedor, Phys. Rev. Lett. 81, 4688 (1998).

[25] K. Schönhammer, O. Gunnarsson, and R. M. Noack, Phys. Rev. B 52, 2504 (1995)

[26] N. A. Lima, M. F. Silva, L. N. Oliveira, and K. Capelle, Phys. Rev. Lett. 90, 146402 (2003).

[27] F. Malet and P. Phys. Rev. Lett. 109, 246402 (2012)

[28] J. Lorenzana, Z.-J. Ying, and V. Brosco, Phys. Rev. B 86, 075131 (2012).

[29] A. Mirtschink, M. Seidl, and P. Gori-Giorgi, Phys. Rev. Lett. 111, 126402 (2013)

[30] C. Verdozzi, Phys. Rev. Lett. 101, 166401 (2008)

[31] S. Kurth, G. Stefanucci, E. Khosravi, C. Verdozzi, and E. K. U. Gross, Phys. Rev. Lett. 104, 236801 (2010)

[32] A.-M. Uimonen, E. Khosravi, A. Stan, G. Stefanucci, S. Kurth, R. van Leeuwen, and E. K. U. Gross, Phys. Rev. B 84, 115103 (2011).

[33] A. Kartsev, D. Karlsson, A. Privitera, and C. Verdozzi, Sci. Rep. 3, 2570 (2013).

[34] M. J. P. Hodgson, J. D. Ramsden, J. B. J. Chapman, P. Lillystone, and R. W. Godby, Phys. Rev. B 88, 241102 (2013).

[35] M. J. P. Hodgson, J. D. Ramsden, and R. W. Godby, Phys. Rev. B 93, 155146 (2016).

[36] G. Stefanucci and S. Kurth, Nano Lett. 15, 8020 (2015)

[37] S. Kurth and G. Stefanucci, Phys. Rev. B 94, 241103(R) (2016).

[38] S. Kurth and G. Stefanucci, J. Phys.: Condens. Matter 29, 413002 (2017)

[39] C. Ullrich, Time-Dependent Density-Functional Theory (Oxford University Press, Oxford, 2012).

[40] N. T. Maitra, J. Chem. Phys. 144, 220901 (2016).

[41] S. Kurth, G. Stefanucci, C.-O. Almbladh, A. Rubio, and E. K. U. Gross, Phys. Rev. B 72, 035308 (2005).

[42] N. Dittmann, J. Splettstoesser, and N. Helbig, arXiv:cond-mat/1706.04547 (2017).

[43] A.-P. Jauho, N. S. Wingreen, and Y. Meir, Phys. Rev. B 50, 5528 (1994)

[44] Y. Goldin and Y. Avishai, Phys. Rev. Lett. 81, 5394 (1998).

[45] Y. Goldin and Y. Avishai, Phys. Rev. B 61, 16750 (2000)

[46] J. M. Elzerman, S. De Franceschi, D. GoldhaberGordon, W. G. van der Wiel, and L. P. Kouwenhoven, J. Low Temp. Phys. 118, 375 (2000). 